\title{
Adenosine Deaminase Levels in Cerebrospinal Fluid in Different Etiology of Meningitis
}

\author{
Amit Bhaskar ${ }^{1}$, Mohammad Shafat Imam Siddiqui ${ }^{2}$, Kumar Bhaskar ${ }^{1}$, Smitha S. ${ }^{1}$ \\ ${ }^{1}$ Assistant professor, Department of Medicine, Heritage Institute of Medical Sciences, Varanasi, ${ }^{2}$ Prof. and HOD, Department of Medicine, Heritage Institute \\ of Medical Sciences, Varanasi
}

\section{Abstract}

Background: Meningitis is a medical emergency as it can be life threatening because of the inflammation proximity to brain and spinal cord. If not recognized earlier, it can lead to serious long term neurological sequelae like deafness, epilepsy, hydrocephalus and cognitive deficits. Subjects and Methods: The study included 75 patients of meningitis, on the basis of clinical findings, CSF findings and MRI brain who were admitted to the emergency and ICU of the hospital. Results: There were $63 \%$ male and $37 \%$ females in TBM $73.9 \%$ male and $26.1 \%$ females in PM and $72 \%$ male and 28\% females in VM group. Conclusion: CSF ADA is markedly raised in tubercular meningitis, while lower ADA values are seen in pyogenic meningitis and viral meningitis.

Keywords: CSF-Adenosine deaminase, Differential Diagnosis of Meningitis, Tuberculous meningitis, Viral meningitis \& Pyogenic meningitis.

Corresponding Author: Dr. Kumar bhaskar, Assistant professor, Department of Medicine, Pearl --21, Royal Residency, Sheel Nagar, Mahmoorganj, Varanasi, UP -221010.

Received: November 2019

Accepted: December 2019

\section{Introduction}

Meningitis is inflammation of the meninges (Pia, Arachnoid and Dura mater) covering the brain and spinal cord. The most common cause of meningitis is infections due to viruses, bacteria, mycobacterium, fungi and other microorganisms. The non-infectious causes include malignancy, chemical compounds, drugs and inflammatory conditions like CNS sarcoidosis. SLE, Behcet's syndrome etc. Meningitis can also be classified according to the temporal profile as acute, sub-acute and chronic types. ${ }^{[1]}$ Meningitis is a medical emergency as it can be life threatening because of the inflammation proximity to brain and spinal cord. If not recognized earlier, it can lead to serious long term neurological sequelae like deafness, epilepsy, hydrocephalus and cognitive deficits. ${ }^{[2]}$ Lumbar puncture and CSF analysis are used to diagnose or exclude meningitis. Management involves prompt administration of antibiotics, and in appropriate situations antiviral and anti tuberculous agents according to the organism suspected. Steroids are used as adjunctive agent to prevent the complications from overactive inflammation. ${ }^{[3]}$ The early diagnosis and treatment remains a challenge to the clinician.The classic triad of diagnostic signs consists of nuchal rigidity, sudden high fever, and altered mental status; however, all the three features are present in only $44-46 \%$ of bacterial meningitis cases. ${ }^{[4]}$

In tuberculous meningitis, the early manifestations are usually low-grade fever, malaise, headache, slow mentation, confusion, and neck rigidity, with Kernig and Brudzinski signs. The seeding of tuberculous bacilli into CSF leads to development of clinical picture comprising of gradually fluctuating fever, weight loss, headache, behaviour changes and vomiting. Interventional delay leads to neurological deficits, loss of consciousness, seizures and it is the most common point of time when the diagnosis of tubercular meningitis is considered. Signs of cranial nerve involvement (usually ocular palsies, less often facial palsies or deafness) and papilledema may be present in TB Meningitis. Viral meningoencephalitis, and particularly herpes simplex virus (HSV) encephalitis, can mimic the clinical presentation of bacterial meningitis. The typical CSF profile with viral CNS infections is a lymphocytic pleocytosis with a normal glucose concentration, in contrast to neutrophilicpleocytosis and hypoglycorrhachia, which is characteristic of bacterial meningitis. ${ }^{[5]}$ Partially treated patients with the use of inappropriate antibiotics can obscure the cytological and biochemical status of the spinal fluid and the chance of recovery of organisms from the spinal fluid will also become less. They can also simulate the CSF picture of viral meningoencephalitis. In Enteroviral meningitis, the polymorphs can flood the CSF in first 6 hours along with normal CSF glucose. In immunosuppressed patients, the mounting of CSF inflammatory response is suboptimal and can also simulate the viral picture. Hence a quick and reliable method for differentiating bacterial and viral meningitis is essential for optimal management outcome. 
Adenosine deaminase (ADA) is an enzyme in the purine salvage pathway that catalyzes the conversion of adenosine and deoxyadenosine to inosine and deoxyinosine respectively with the release of ammonia. It plays important role in differentiating lymphoid cells and is present in abundance in active T-lymphocytes whose concentration is inversely proportional to the degree of differentiation. ${ }^{[6]}$ Its levels are ten times higher in T-lymphocytes than in erythrocytes. The enzyme activity increases during mitogenic and antigenic responses of lymphocytes and Tlymphocyteblastogenesis can be inhibited by inhibitors of ADA. ADA is now being recognized as a marker of cell mediated immunity particularly as a marker of $\mathrm{T}$ lymphocyte activation. Adenosine deaminase levels (ADA) have also been considered by several researchers to differentiate tubercular disease from non-tubercular. ${ }^{[7,8]}$ This present study was aimed to differentiate different types of meningitis o basis of etiologies including pyogenic, tuberculous and viral meningitis using ADA levels in CSF.

\section{Subjects and Methods}

This present study was conducted in the Department of Medicine, Heritage Institute of Medical Sciences, Varanasi during the period 2017 August to September 2019. The study included 75 patients of meningitis, on the basis of clinical findings, CSF findings and MRI brain who were admitted to the emergency and ICU of the hospital. All those Patients who were diagnosed with meningitis and meningoencephalitis were included in the study.

\section{Exclusion criteria}

- Patient already on treatment of meningitis

- Patients with acute infection at sites other than CNS.

- Patient in whom lumbar puncture is contraindicated

- Associated sever hepatic dysfunction

- Females on oral contraceptives and intrauterine device

- Sever dyslipidemia and patients on steroid treatment.

Biochemical testing was performed in the department of Biochemistry, HIMS, Varanasi. This study was undertaken after approval of the Institutional Ethics Committee, HIMS, Varanasi. A Written informed consent of the patients was obtained in vernacular language for their inclusion. Complete clinical examination and proper detailed history of all the meningitis patients admitted in medical wards was recorded, at the time of admission. Finally, the clinical evaluation was observed and recorded. According to the above mentioned criteria patients were diagnosed as Tuberculous, Pyogenic and Viral Meningitis.

Estimation of ADA in CSF: Turbidimetry method was used for ADA estimation.

\section{Statistical analysis:}

The data was collected systematically and analysed statistically according to the standard statistical methods using One way analysis of variance (ANOVA) and $p$ value $<0.005$ was considered as significant.

\section{Results\& Discussion}

In the present study total of 75 patients of clinically suspected meningitis were included of which there were 27 cases of Tubercular Meningitis, 23 cases of Pyogenic Meningitis and 25 cases of Viral meningitis.

Mean age of subjects in our study came out to be $39.4 \pm 12.2$ years with majority of subjects (29\%) belonging to the age group of 30-39 years. Male predominance was observed comprising of $69.3 \%$ males and overall, Male: female ratio in the present study observed was 2.26:1. Maximum male population was seen in age range of 20-29 years while maximum female population was seen in 30-39 and 50-59 years of age.

Table 1: Clinical presentation of patients with meningitis

\begin{tabular}{|l|l|}
\hline Clinical symptoms and signs & No. of patients(N=75) \% \\
\hline Fever & $40(53.3 \%)$ \\
\hline Headache & $36(48 \%)$ \\
\hline Nausea and vomiting & $33(44 \%)$ \\
\hline Kernig's sign & $56(74.7 \%)$ \\
\hline Neck rigidity & $61(81.3 \%)$ \\
\hline Altered sensorium & $16(21.3 \%)$ \\
\hline Focal neurological deficit & $22(29.3 \%)$ \\
\hline Seizure(s) & $10(13.3 \%)$ \\
\hline
\end{tabular}

[Table 1] shows the common clinical presentation of patients with meningitis in our study were: neck rigidity $(81.3 \%)$, positive Kernig's sign $(74.7 \%)$, fever $(53.3 \%)$, headache $(48 \%)$, nausea and vomiting (44\%) while Few patients of meningitis were having focal neurologic deficit, altered sensorium, and seizures.

Table 2: Gender wise distribution in cases of meningitis.

\begin{tabular}{|l|l|l|l|}
\hline Sex & TMB (27) & PM(23) & VM(25) \\
\hline Male & $17(63.0 \%)$ & $17(73.9 \%)$ & $18(72.0 \%)$ \\
\hline Female & $10(37.0 \%)$ & $06(26.1 \%)$ & $07(28.0 \%)$ \\
\hline
\end{tabular}

[Table 2] shows gender wise distribution in meningitis cases. There were $63 \%$ male and $37 \%$ females in TBM $73.9 \%$ male and $26.1 \%$ females in PM and $72 \%$ male and $28 \%$ females in VM group. Overall male case were more in number compared to female in our study however there were no statistically significant difference seen $(\mathrm{p}>0.05 \%)$.

Table 3: CSF: ADA Levels and Diagnosis in Different Types of Meningitis

\begin{tabular}{|l|l|l|l|}
\hline \multirow{2}{*}{ Diagnosis } & \multicolumn{2}{|l|}{ ADA Levels (IU/L) } & \multirow{2}{*}{ P Value } \\
\cline { 2 - 3 } & Mean \pm S. D. & Min- Max & \\
\hline TMB & $17.31 \pm 4.07$ & $7.9-25.8$ & \multirow{3}{*}{$\mathrm{P}<0.001$} \\
\hline PM & $3.65 \pm 1.34$ & $1.7-8.2$ & \\
\hline VM & $3.16 \pm 1.23$ & $1.2-9.1$ & \\
\hline
\end{tabular}

[Table 3] shows the ADA levels in different types of meningitis as observed showed that ADA levels in TBM were in the range of 7.9- $25.8 \mathrm{IU} / \mathrm{l}$ with mean of $17.31 \pm 4.07$ IU/l, In pyogenic meningitis were 1.7-8.2 IU/l with mean $3.65 \pm 1.34 \mathrm{IU} / 1$ while in viral meningitis were of 1.2-9.1 IU/l with mean of $3.16 \pm 1.23 \mathrm{IU} / 1$. On comparison, CSF ADA was found to be markedly raised in TB meningitis, while lower ADA values are seen in PM and VM. Hence, difference 
between ADA values between TBM \& PM and TBM \& VM in both the groups is statistically significant $(\mathrm{p}<0.001)$. Estimation of CSF ADAhas been considered as a marker of cell-mediated immunity and its activity has been observed in various infections including TBM. Routine CSF laboratory findings may not be helpful to differentiate tuberculous etiology in meningitis from other causes. Considering that both humoral and cellmediated immunity play an important role in TBM infection, it has been suggested that ADA activity in CSF may help differentiate TBM from non-TBM infectious meningitis. Therefore in the present study we aimed to differentiate different types of meningitis o basis of etiologies including pyogenic, tuberculous and viral meningitis using ADA levels in CSF. The mean ADA activity was $17.31 \pm 4.07 \mathrm{U} / 1$ in the tubercular meningitis group, $3.65 \pm 1.34$ in pyogenic meningitis group and $3.16 \pm 1.23$ in the viral meningitis group. The results of our study showed that on comparison, CSF ADA is markedly raised in TB meningitis, while lower ADA values are seen in Pyogenic Meningitis and Viral Meningitis. On comparison, difference between ADA values between TBM \& Pyogenic Meningitis and TBM \& Viral Meningitis in both the groups is statistically significant. In accordance to our results, previous studies done by TuonFFet al, ${ }^{[9]}$ Moghtaderi A et al, ${ }^{[10]}$ Gupta et al, ${ }^{[11]}$ Agarwal $S,{ }^{[12]}$ Gautam et al, ${ }^{[13]}$ Belagavi and Shalini, ${ }^{[14]}$ and Mehta et al, ${ }^{[15]}$ had shown that ADA levels were found to be significantly higher in TBM group as compared to bacterial and viral meningitis. On the contrary, Abdolbagi $\mathrm{MH}$ et al, ${ }^{[16]}$ reported that the measurement of ADA level alone is not useful as a rapid diagnostic test for TBM. Possible explanation for raised ADA levels can be because ADA has been considered as a marker of cell mediated immunity as it is released by $\mathrm{T}$ cells during cell mediated immune response (CMI) to the tubercle bacilli. Its activity has been observed in various infections including TBM. Considering that both humoral and cell-mediated immunity play an important role in TBM infection, it has been suggested that ADA activity in CSF may help differentiate TBM from non- TBM infectious meningitis. Gupta et al, ${ }^{[11]}$ observed that adenosine deaminase levels in nontuberculous disease rarely exceeded the cut-off; set for tuberculous disease. They have further observed that ADA estimation is not only a fairly sensitive and specific test (more than $94 \%$ ), helpful in differentiating tubercular from non-tubercular etiology; both in pulmonary and extrapulmonary disease but is also simple, inexpensive and rapid. For this reason this test may help in early diagnosis, improve the prognosis and reduce spread of disease and sequlae. ${ }^{[17]}$ Chander A et al, ${ }^{[18]}$ reported that if the ADA assay result is negative, the probability that the patient has TBM is approximately $19 \%$, which is not low enough to rule out TBM. Thus they suggest, that a negative ADA assay result should not be used alone as a justification to exclude or discontinue anti-TB treatment. The choice of therapeutic strategy should be based on the results of microscopic examination of a smear or culture for Mycobacterium tuberculosis, as well as other clinical data, such as response to anti-TB treatment. Similarly, Choi SH et al studied ADA activity in CSF of 182 patients with meningitis. The mean ADA level in tubercular group was $12.7 \pm 7.5 \mathrm{U} / 1$ and it was significantly higher than the other groups. ${ }^{[19]}$ Chotmongkol V et al identified a CSF ADA level of $15.5 \mathrm{U} / 1$ as the best cutoff value to differentiate tubercular meningitis and nontubercular meningitis, with sensitivity of $75 \%$ and specificity of $93.0 \%$.[20] So we concluded that CSF-ADA level can help in the differentiation of tubercular meningitis from nontubercular meningitis. However, they should be interpreted judiciously in the light of the patients' clinical manifestations and the CSF physical, biochemical and microscopic characteristics.

\section{Conclusion}

CSF ADA is markedly raised in tubercular meningitis, while lower ADA values are seen in pyogenic meningitis and viral meningitis. Hence, we can suggest that estimation of these enzymatic activities can be helpful in diagnosing and differentiating pyogenic, tubercular and viral meningitis. So it can be used as simple rapid inexpensive test for differentiating tubercular from pyogenic and viral meningitis.

\section{References}

1. A Adams and R.A. Harkness. A Brief Communication: ADA activity in thymus and other human tissues. Clin. Exp. Immunol 1976; 26: 647 649

2. Abe C, Hirano K, Wada M, Kazumi Y, Takahashi M, Fukasawa Y, Yoshimura T, et al. Detection of Mycobacterium tuberculosis in clinical specimens by polymerase chain reaction and Gene probe Ampli $\square$ ed Mycobacterium Tuberculosis Direct Test. J ClinMicrobiol 1993; 31 (12): 3270-3274

3. Allan H. Rooper, Martin A Samuels. Adams and Victor's Principles of Neurology. 9th ed. The McGraw-Hill Companies, Inc. 2009;32:668-690 Infections of the Nervous System and Sarcoidosis and 2009;33:712-713 Viral Infections of the Nervous System, Chronic Meningitis and Prion Diseases.

4. Van de Beek D, De Gans J, Spanjaard L, Weisfelt M, Reitsma JB, Vermeulen M. Clinical features and prognostic factors in adults with bacterial meningitis. New Eng J Med. 2004;351(18):1849-59.

5. Roos KL, Tyler KL. Meningitis, Encephalitis, Brain abscess, and Empyema. In Harrison's Principles of Internal Medicine. 16th ed. NewYork: McGraw-Hill Professional; 2004. p.2471-90

6. Sharma SK, Mohan A. Adenosine deaminase in the diagnosis of tuberculosis pleural effusion. Indian $J$ Chest Dis Allied Sci. 1996;38(2):69-71.

7. Malan C, Donald PR, Golden M, Taljaard JJ. Adenosine deaminase levels in cerebrospinal fluid in the diagnosis of tuberculous meningitis. $\mathbf{J}$ Trop Med Hyg. 1984;87(1):33-40

8. Gupta B K, Bharat Vinay, BandyopadhyayDebapriya. Sensitivity, specificity, negative and positive predictive values of Adenosine deaminase in patients of Tubercular and Non-Tubercular Serosal Effusion in India. J Clin Med Res. 2010 Jun;2(3):121-126.

9. Tuon FF, Higashino HR, Lopes I, Litvoc MN, Atomiya AN, Antonangelo L, et al. Adenosine deaminase and tuberculous meningitisA systematic review with meta-analysis. Scand $J$ Infect Dis. 2010;42:198-207.

10. Moghtaderi A, Niazi A, Alavi-Naini R, Yaghoobi S, Narouie B. Comparative analysis of cerebrospinal fluid adenosine deaminase in tuberculous and non-tuberculous meningitis. ClinNeurolNeurosurg. 2010;112:459-62.

11. Gupta BK, Bharat A, Debapriya B, Baruah H. Adenosine deaminase levels in CSF of tuberculous meningitis patients. J Clin Med Res. 2010;2(5):220-4.

12. Agarwal S. Study of adenosine deaminase activity as a biochemical marker of cell mediated immunity in tuberculous meningitis, tuberculous pleural effusions and tuberculous ascites. J Medicine. 2012;13:32-38.

13. Gautam N, Aryal M, Bhatta N, Bhattacharya SK, Baral N, Lamsal M. 


\section{Bhaskar et al: Adenasine Deaminase Levels in Cereleraspinal Fluid}

Comparative study of cerebrospinal fluid adenosine deaminase activity in patients with meningitis. Nepal Med Coll J. 2007;9(2):104-6.

14. Belagavi AC, Shalini M. Cerebrospinal fluid C reactive protein and adenosine deaminase in meningitis in adults. JAPI. 2011;59:557-60.

15. Mehta M, Shah J, Leuva AT. CSF ADA as diagnostic tool for tuberculous meningitis patients. NJIRM. 2012;3(3):160-4.

16. Abdolbagi MH, Dezfuli AR, Soudbakhsh AR, Rasulinejad M, Golestani B. Diagnostic evaluation of adenosine deaminase in cerebrospinal fluid in tuberculous meningitis. Kanun Med J. 2006;8(4):23-8.

17. Giusti G. Adenosine deaminase. In: Bergmeyer H.U., editor. Method of enzymatic analysis. II. Florida: VCH Weinheim; 1974. p. 1072.
18. Chander A, Shrestha CD. Cerebrospinal fluid adenosine deaminase levels as a diagnostic marker in tuberculous meningitis in adult Nepalese patients. Asian Pac J Trop Dis. 2013;3(1):16-9.

19. Choi SH, KimYS, Bae IG, Chung JW, Lee MS, Kang JM, Ryu J, Woo $\mathrm{JH}$. The possible role of cerebrospinal fluid adenosine deaminase activity in the diagnosis of tubercular.

20. Chotmongkol V, Teerajetgul GY, Yodwut C. Cerebrospinal fluid adenosine deaminase activity for the diagnosis of tubercular meningitis in adults. Southeast Asia J Trop Med Public Health. 2006;37:948-52.

Copyright: () the author(s), 2019. It is an open-access article distributed under the terms of the Creative Commons Attribution License (CC BY 4.0), which permits authors to retain ownership of the copyright for their content, and allow anyone to download, reuse, reprint, modify, distribute and/or copy the content as long as the original authors and source are cited.

How to cite this article: Bhaskar A, Siddiqui MSI, Bhaskar K, Smitha S. Adenosine Deaminase Levels in Cerebrospinal Fluid in Different Etiology of Meningitis.Acad. J Med. 2019;2(2):186-89.

DOI: dx.doi.org/10.21276/ajm.2020.2.2.48

Source of Support: Nil, Conflict of Interest: None declared. 